\title{
Quantifying Cell-to-Cell Variations in Lithium Ion Batteries
}

\author{
Shriram Santhanagopalan ${ }^{1}$ and Ralph E. White ${ }^{2}$ \\ ${ }^{1}$ National Renewable Energy Laboratory, Golden, CO 80401, USA \\ ${ }^{2}$ University of South Carolina, Columbia, SC 29204, USA \\ Correspondence should be addressed to Shriram Santhanagopalan, shriram.santhanagopalan@nrel.gov
}

Received 26 March 2011; Accepted 4 October 2011

Academic Editor: Dong Shu

Copyright (C) 2012 S. Santhanagopalan and R. E. White. This is an open access article distributed under the Creative Commons Attribution License, which permits unrestricted use, distribution, and reproduction in any medium, provided the original work is properly cited.

Lithium ion batteries have conventionally been manufactured in small capacities but large volumes for consumer electronics applications. More recently, the industry has seen a surge in the individual cell capacities, as well as the number of cells used to build modules and packs. Reducing cell-to-cell and lot-to-lot variations has been identified as one of the major means to reduce the rejection rate when building the packs as well as to improve pack durability. The tight quality control measures have been passed on from the pack manufactures to the companies building the individual cells and in turn to the components. This paper identifies a quantitative procedure utilizing impedance spectroscopy, a commonly used tool, to determine the effects of material variability on the cell performance, to compare the relative importance of uncertainties in the component properties, and to suggest a rational procedure to set quality control specifications for the various components of a cell, that will reduce cell-to-cell variability, while preventing undue requirements on uniformity that often result in excessive cost of manufacturing but have a limited impact on the cells' performance.

\section{Introduction}

Lithium ion batteries for consumer electronics applications such as laptop computers and camcorders have been manufactured in the millions every month [1]. A typical scrap rate of a few percentages, while has been frowned upon, has not been identified as a major impediment to the use of this technology. The typical life expectation of these cells has been three years and most applications use up to six cells in a series/parallel configuration; the operating scenario for these cells has also been mild to moderate-for example, most laptop applications do not require the cells to discharge at a rate higher than $1 \mathrm{C}$ (i.e., most laptops can operate for one hour or more when powered by their batteries only) and usually operate at moderate temperatures. Consequently, the quality requirements on batteries have been considerate, compared to those on other electronic components. With the advent of large format batteries for vehicle and smartgrid applications, the requirements on cell standards have grown exponentially (e.g., compare safety standards IEEE 1625 and Electric and Hybrid Vehicle Propulsion Battery System Safety Standard, SAE J2929 [2]). The size of the individual cells has increased at least three fold; the cost to build the cells has increased with their capacities and so has the need to reduce the rejection rates. Similarly, the number of cells packaged to build modules and packs for such large applications has increased as well, and the need to ensure the longer life time (e.g., ten years for battery packs used in vehicles) and to guarantee performance under harsh operating conditions demands better uniformity among the individual cells. Standard practices in the automotive industry such as Advanced Product Quality Planning (APQP) and Production Part Approval Process (PPAP) are now typical of cell manufacturersright from raw material purchase until product testing and acceptance of the component by the OEMs. The lithiumion chemistry has a stringent intolerance to impurities and a high requirement for uniformity at the component level. Nevertheless, the cell manufacturing process is subtly different from the manufacturing of other electronics: for example, the various functional components such as the electrolyte and separator are usually manufactured at different locations, and these components themselves contain multiple ingredients supplied by different vendors. Quality 
control has been emphasized at each stage-but rarely is there a coordinated effort to quantify variations in product specifications and how variations among the different components interact. Despite efforts by various battery manufacturers to develop in-house capabilities to produce all components at one location, limited know-how on product handling has often resulted in limited improvements. Overall, battery manufacturing has, at best, remained an art even after two decades.

The most emphasis on enhancements to batteries for transport applications today is on the cost. The missing link between the requirements for the pack and the specifications for the individual cells is currently addressed by over-sizing the battery packs. A battery electric vehicle utilizes 30-50\% of the available capacity in its battery to allow for longevity of the packs as well as to ensure safe operation of the battery. Reducing the size of the battery packs has been identified as a major stepping stone for long-term viability of battery-powered vehicles. Immediate efforts in place include rigorous characterization of the packs by the end-users and the OEMs; requirements on battery performance and durability have been arrived at based on voluminous drivecycle testing, for example. Such requirements translate to tighter quality control measures all the way down to the rawmaterial selection. While it is important to ensure quality of the cells, it is common knowledge that variations in some materials are naturally larger than in others, and that some properties influence the performance of the cell more than others. The allowable range of uncertainty in material properties has by and large been determined on an ad-hoc basis, sometimes demanding for higher standards than necessary, resulting in added cost of manufacturing, while in other cases, the variations in material property have not been identified as critical until after extensive design of experiment studies and/or costly field incidents. Most component manufacturers collect variability in the key properties and distribute this information to the cell manufacturers. Similarly, most cell manufacturers share information about the cell-to-cell variability limits with the OEM's. A systematic approach to relate uncertainty at the material level to the cell-to-cell variations will greatly help reduce the rejection rate of the cells and prevent undue quality requirements at the component level-both leading to significant enhancement in the performance and cost of battery packs.

In this work, we demonstrate utilizing a mathematical model in the impedance domain, the correlation between the variations in material properties, and the performance at the cell level, outlining a procedure to compare the relative importance of uncertainties in the component properties and to suggest a rational procedure to set quality control specifications for the various components of a cell. The methodology presented here can be utilized as a screening test (customizable by the cell manufacturer) to ascertain quality requirements for the various components and typically involves measuring the standard a.c. impedance response at a predetermined frequency(ies) - similar to the cell internal resistance measurement used currently in the industry.

\section{Mathematical Model}

The choice of impedance spectroscopy as the screening tool provides the flexibility for use without introducing significant perturbation to the cell, from the requisite operating protocol and results in minimal changes to the cell characteristics. So, this technique has been employed extensively in the past to investigate response of electrochemical systems [3]. Several mathematical models for the impedance response of lithium ion batteries exist in the literature [4-11]. Whereas the most common approach to interpreting impedance response is utilizing empirical equivalent circuits [3]; for the purpose of identifying critical material components and their relative impact on the cell performance, a physics-based model is preferable. The model developed by Darling and Newman [4] utilizes the porous electrode theory to account for the effect of parameters like porosity, particle size distribution, and thickness of the electrode as well as properties of the electrolyte on the cell impedance. This model also captures the surface effects on the kinetics as well as the dependence of the thermodynamics on the chosen chemistry. Dees et al. [6] used a similar model to characterize surface modifications on the cathode. Since then, Meyers et al. [5] and Methekar et al. [11] have developed closed-form solutions under limiting cases that allow one to interpret the physical significance of the impedance measurements at various frequencies. More recently, comprehensive models to simulate the performance of a lithium ion cell containing two intercalation electrodes and a porous separator have been developed by Sikha and White $[8,9]$ and Motupally et al. [12].

In the present work, we utilize the results of Sikha and White $[8,9]$ to simulate the impedance response of the cell. The use of this model has a few advantages: the contributions from the individual components to the overall cell impedance are readily recognizable; the closedform transfer function for the lithium ion cell allows us to use the conventional tools [13-23] in controls theory to investigate effects of uncertainty in the parameters on the cell performance. Further details on the model equations can be found in $[8,9]$. A list of base parameters used in the present work is summarized in Table 1 [9]. As discussed in subsequent sections, these parameters are perturbed to reflect the variations during the manufacturing process and their relevance to the cell performance. The cell model incorporating the base-line parameters is referred to as the "nominal" cell model in the discussion.

The cell impedance was evaluated between $1 e-6$ and $1 e-$ $4 \mathrm{~Hz}$, using the closed form solution presented by Sikha, using Matlab (C). For an elaborate description of the versatility of the model and its applicability under various limiting conditions, the reader is referred to the original work; it is worth mentioning that sufficient care must be taken to ensure numerical precision as addressed in the earlier efforts $[5,9]$. The entire frequency range was evenly divided in the log space into 1000 frequencies. The results shown are for a cobalt oxide cathode, a polypropylene separator, and a graphitic anode. 
TABLE 1: List of nominal parameters used in the impedance model.

\begin{tabular}{lcc}
\hline Parameter & Cathode & Separator \\
\hline Diffusion coefficient in the solid particles $\left(\mathrm{m}^{2} / \mathrm{s}\right)$ & $1 e-12$ & -10 \\
Solid phase conductivity $(\mathrm{S} / \mathrm{m})$ & 10 & 0.45 \\
Nominal porosity & 0.15 & 100 \\
Filler fraction & 0.5 & 0.4382 \\
Transfer coefficient & 8.5 & 0.0566 \\
Nominal particle size $(\mu \mathrm{m})$ & 10 & 0.5 \\
Equilibrium exchange current density $\left(\mathrm{A} / \mathrm{m}^{2}\right)$ & 0.2 & 12.5 \\
Double layer capacitance for the electrode $\left(\mathrm{F} / \mathrm{m}^{2}\right)$ & 0.02 & 10 \\
Double layer capacitance for the surface film $\left(\mathrm{F} / \mathrm{m}^{2}\right)$ & $1 e-4$ & 0.2 \\
Resistance for the surface film $\left(\mathrm{Ohm}-\mathrm{m}^{2}\right)$ & 2.3 & 1.5 \\
Tortuosity & 70 & 25 \\
Nominal electrode thickness $(\mu \mathrm{m})$ & $0.99-0.52$ & $5 e-4$ \\
Stoichiometric window & & 4.1 \\
Initial concentration of the electrolyte $(\mathrm{M})$ & & 73.5 \\
Conductivity of the electrolyte $(\mathrm{S} / \mathrm{m})$ & & $0.7952-0.01$ \\
Diffusivity in the electrolyte $\left(\mathrm{m}^{2} / \mathrm{s}\right)$ & & $2.58 e-10$ \\
Transport number & & 0.435 \\
Nominal room temperature & & $298 \mathrm{~K}$
\end{tabular}

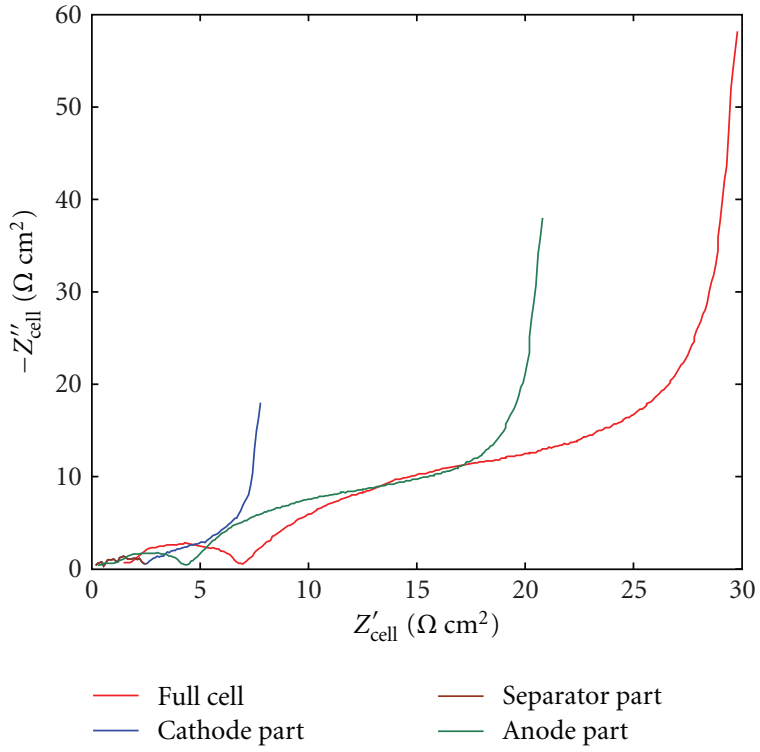

FIGURE 1: Nyquist plot showing the contribution of the individual cell components towards the overall impedance of the cell at a full cell SOC value of 0.5 , between $10^{6}$ and $10^{-4} \mathrm{~Hz}$.

\section{Results and Discussion}

Figure 1 presents the Nyquist response for the nominal cellthe results presented are similar to the $50 \%$ state of charge (SOC) case presented by Sikha and White [9]. The model captures the low-frequency diffusion tail followed by the solution phase transport features, charge transfer, and double layer effects and finally the ohmic drop with increasing range of frequencies. Also shown is the impedance of the individual components-namely, the anode, cathode, and the separator. For the nominal cell, the contributions from the separator were found to be negligible; the anode and cathode responses were similar in shape: each curve showing the characteristic features described above.

3.1. Incorporating Distributed Parameters. Earlier efforts to incorporate distribution in material properties, have considered model electrodes fabricated to include several characteristic particle sizes. For example, Darling and Newman [4] illustrated that the coloumbic capacity of the nonuniform electrodes was consistently lower than the predictions made from a model utilizing a single particle size, at a prescribed galvanostatic discharge rate. The results were shown to progressively worsen at higher rates and with increasing particle nonuniformity. Hence, models which presume a constant particle radius were inferred to show the poorest agreement with experimental results at high discharge rates. Stephenson et al. [7] extended these results to incorporate six characteristic particle sizes. In a similar work, Nagarajan et al. [10] demonstrate that an electrode comprised of particles of two different sizes can have a significantly higher capacity than an electrode consisting of single-size particles and that a trade-off between packing density and liquid-phase diffusion resistance can be achieved by adjusting the particle size, volume fraction of large and small particles, and the size ratio to optimize discharge capacity under different operating scenario. Wang and Srinivasan [24] introduced a direct numerical simulation technique that can incorporate a continuous variation in particle size. More recently, Meyers et al. [5] implemented an impedance model that represents the particle radius using a characteristic length obtained by integrating the particle size distribution. We presented a method- 

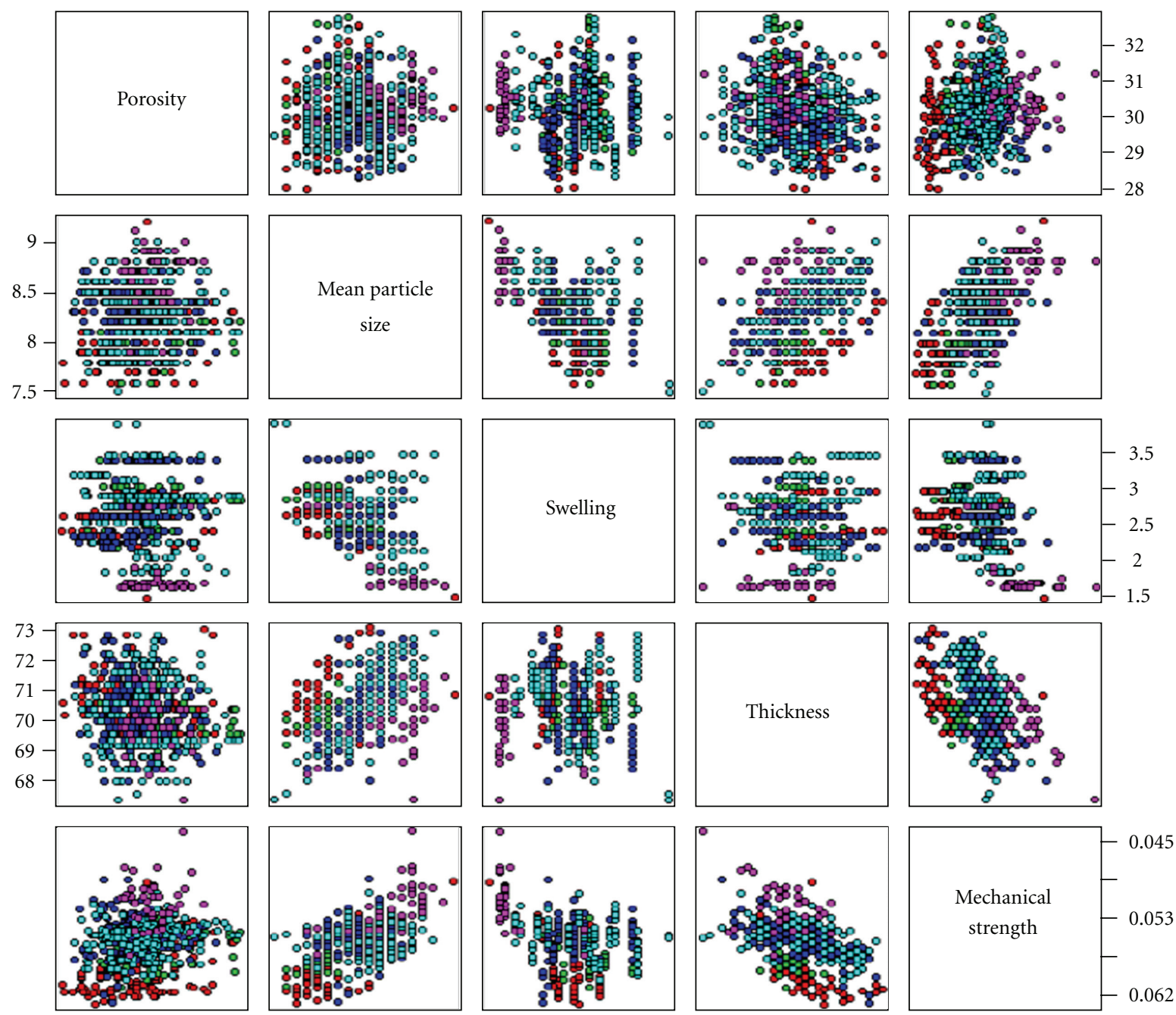

FIGURE 2: Interdependence of five battery material properties: the different colors indicate cells made from five different batches; the spread in each box shows how different the codependence of each pair of properties can be from another. For example, moving across the last column, the mechanical strength and porosity show a weaker correlation, compared to mechanical strength and thickness.

ology to incorporate distributed parameter values into simple battery models using the polynomial chaos approach [14]. However, the limitations on the complexity of the battery model did not allow for extension of the technique to applications beyond system identification. Dubarry et al. [25] analyzed the differences in cell capacity and the weight distribution in 100 cells using an equivalent circuit model and related these issues to the intrinsic cell imbalance to improve the fidelity of battery pack simulations when cellto-cell variations were taken into consideration. The authors quantified the rate and SOC dependence of the polarization resistance in the cells to understand the effect of cell-tocell variations on performance and at the pack level. While presenting a significant improvement over the prior work, the method presented in Dubarry's work could not be used to compare variations from one cell component versus another, largely due to the choice of a simple battery model. Other efforts include models that model nonuniformity in electrodes using Latin hypercubes for sample spacing [26] and
Kinetic Monte Carlo Simulations [11]. Very few of these models incorporate features to address the interaction of different parameters and are hence limited in their practical applicability to address cell quality.

Figure 2 shows the distribution of five different parameters and their codependence on each other. For example, these parameters, A through E, may indicate the porosity, thickness, the mean particle-size, swelling, and mechanical strength of the electrode respectively. The different colors indicate different batches of the product. Two observations are apparent from the data: (i) the parameters specified by the manufacturer to characterize a component are interdependent on each other and (ii) the dependence of a given parameter (e.g., Mechanical Strength) upon one property (e.g., Mean Particle Size) is drastically different from that on another (e.g., Thickness). Failure to comprehend these results often leads to design of experiments focused on optimizing parameters one at a time and does not lead to fruition. In the next few sections, we present a methodology to 


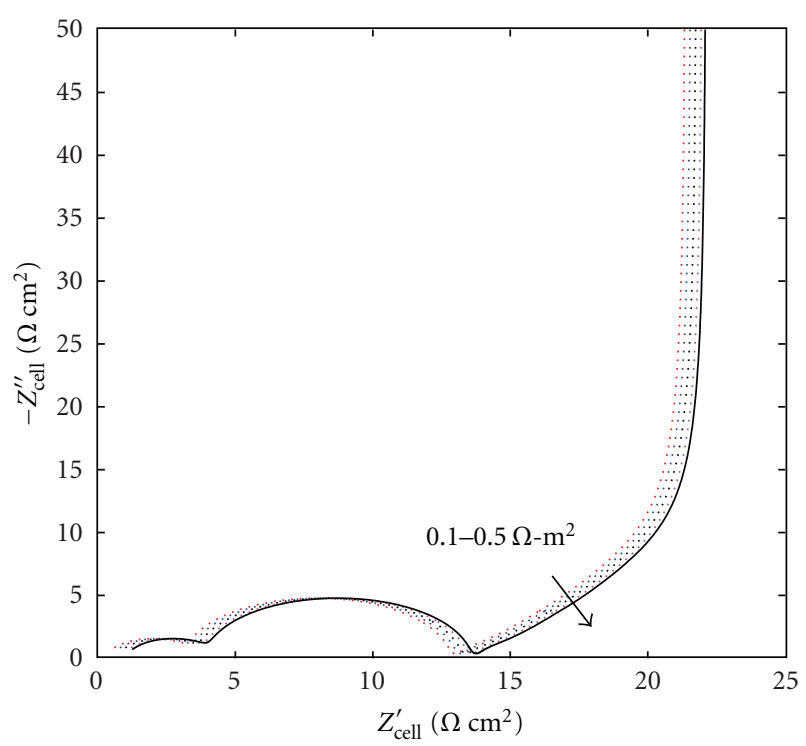

Figure 3: Effect of contact resistance on the impedance response of the cathode: the incorporation of an ohmic drop across the terminals shifts the real axis of the nominal response to more positive values.

incorporate such statistical information obtained in the industry to analyze the relative impact of the different parameters on the cell performance.

3.2. Sensitivity Analysis. As mentioned earlier, the advantage of frequency domain analysis is the ability to capture the signature of phenomena exhibiting different time constants using small perturbations about the equilibrium state. According to the difference in the response time, ohmic drop and kinetic processes are often found to alter the high frequency response, while the effect of slower transport processes is observed at the lower frequencies, around the diffusion tail. It is a standard practice to carry out sensitivity analyses to understand the effect of the different parameters. Figure 3 shows the effect of the contact resistance on the impedance response of the cathode, for example, the incorporation of an ohmic drop across the terminals merely shifts the real axis of the nominal response to more positive values. This is a relatively simple result to interpret, both since the resistive component does not have an imaginary component part and because the time constant for a resistor is significantly different from the other processes taking place within the cell.

Figure 4 presents the interaction of the distribution in different parameter values. Four different parameters are chosen for analysis: the cathode thickness, porosity, tortuosity, and the mean particle radius for the cathode. Note that the particle size is represented by a single value in the model by Sikha and White [8]; however, extension to the equivalent characteristic length approach presented by Meyers et al. [5] to include particle size distributions, is straight forward. In each case, the parameter of interest is perturbed from the nominal value shown in Table 1 by the range indicated in the corresponding Figures Figure 4(a) through $4(\mathrm{~d})$ while retaining the other parameters at their nominal values. Note that the range across which the properties are altered is within the range of experimental variations. Some of these parameters have a greater influence on the impedance response, compared to the others; this trend reflects the choice of nominal values for the parameters chosen and should not be interpreted as the norm. Also, whereas some of the parameters are readily controlled during the manufacturing process, others are usually measured at a later stage to ensure quality requirementsfor example, the tortuosity is not preset to a specific value; it is measured after the electrode is fabricated to account for a certain loading, particle size, and thickness. Nevertheless, the effect of tortuosity is independent of that of the other parameters and is significant under the conditions shown in Figure 4. Typically in such cases, the parameter of interest (here, the tortuosity) has a broader distribution than those that can be preset.

Figure 5 compares the effect of variations in similar parameters (thickness, porosity, and tortuosity) between the separator and the cathode. Since changes in these properties both in the cathode as well as in the separator alter transport in the electrolyte, the low medium frequency (e.g., $\sim 1 \mathrm{mHz}$ ) response reflects these perturbations for all cases. It is under situations like these, when there is an overlap in the response, that a physics-based model is valuable to directly correlate the changes in the design parameters within a meaningful range to the variations in the cell performance. As the results indicate, variations in the cathode, in general, have a greater impact than the corresponding changes in the separator, again, for the set of nominal parameters shown in Table 1. However, most of these values are close to actual properties measured from a working cell, and hence, the trend shown in Figures 4 and 5 is in good qualitative agreement with actual measurements, even if not rigorously quantitative. In practice, tuning the properties of one component may not be as flexible as another-for example, it is easier to fabricate thin $(10 \mu \mathrm{m})$ copper foils of uniform thickness since the processing limitations on metallic rolls have been overcome to a greater extent, than when fabricating composite electrodes or polymer membranes of similar thickness. In such cases, imposing the same tolerance limits for the various components results in additional cost due to increase in rejection rate as well as sophistication in material handling. However, as noted from Figure 5, such variations may not be the limiting factors that impact on the performance of the given cell design. In such instances, the overspecification of component requirements only leads to additional cost, without any noticeable impact on quality.

Despite similar results illustrated in the literature, such practices continue in the industry, primarily because of the influence of one parameter on the other, as previously discussed in Figure 2. For example, whereas the separator porosity may not be critical for a given choice of cathodes (e.g., a high energy cell), the use of a similar product for a high rate application, or even in the same cell when operated at a different temperature, may alter the sensitivity plots shown in Figures 4 and 5 . In the next section, we present a methodology to compare the relative significance of the variations introduced due to the uncertainty in the different 


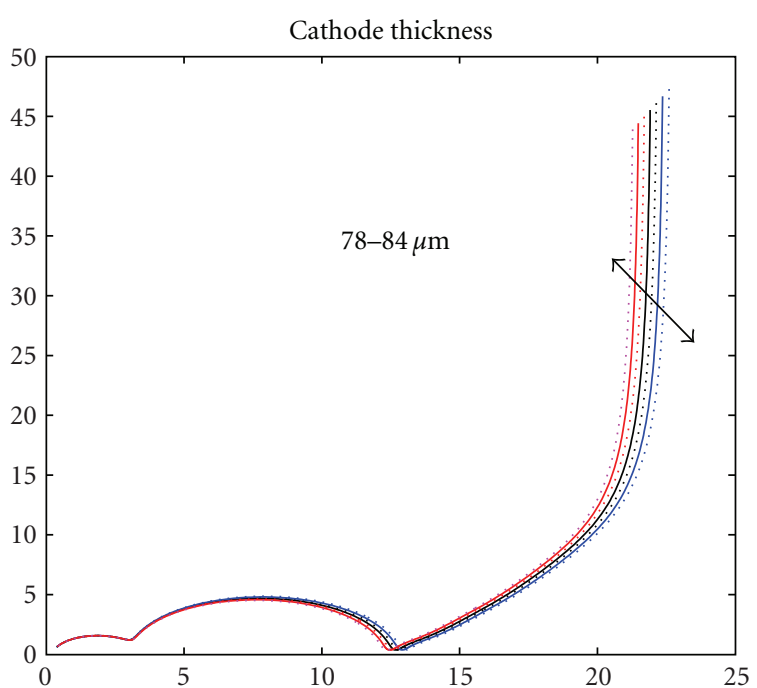

(a)

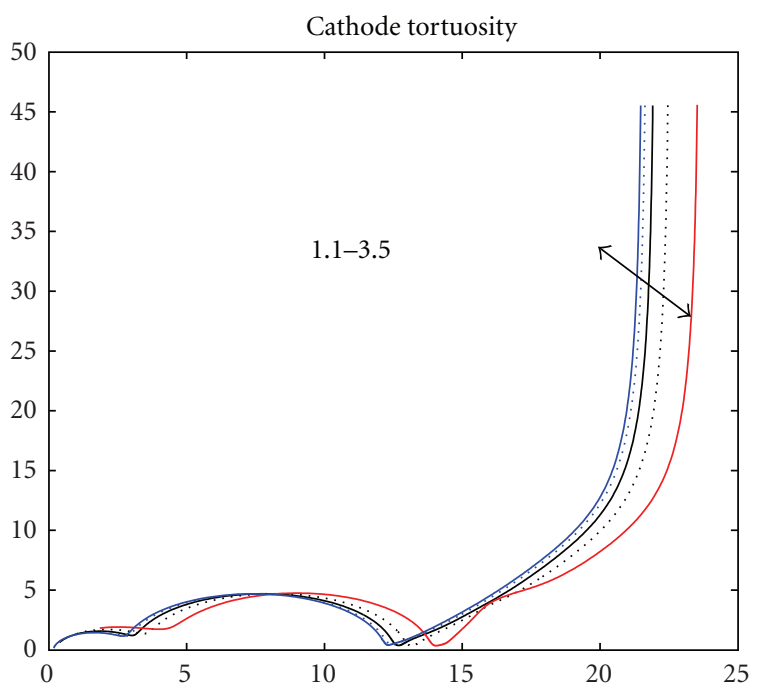

(c)

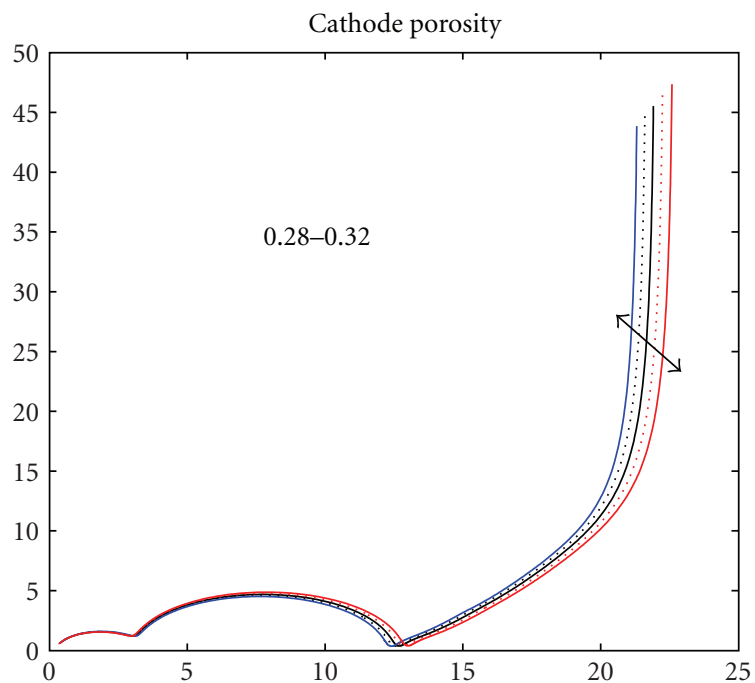

(b)

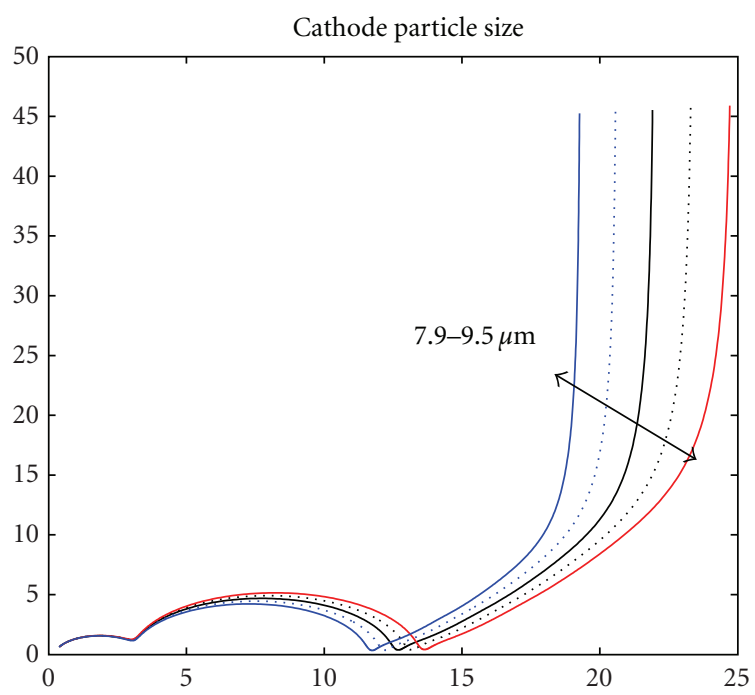

(d)

FIGURE 4: Sensitivity analysis on the cathode: plotted are the Nyquist responses when the design parameters at the cathode are swept about their corresponding nominal values, within the range of manufacturing variability.

parameters to quantify the net impact on the cell as well as to identify the best design solution available to minimize the variations in cell performance.

3.3. Parameter Uncertainty and Process Variability. Most of the discussion presented in this section draws from the literature on robust design under model uncertainties [13-23]. Extensive studies to minimize the effect of uncertainty in the input parameters on the robustness of plant performance have been carried out over several decades [13]. Box converted the correlation matrix into a diagonal form and used the eigenvalues and eigenvectors to define a hyperdimensional ellipse that represents the joint confidence intervals. Routh and Nyquist stability criteria are outlined in standard controls textbooks $[15,23]$. Skogestad and Morari [16] provide a detailed mathematical framework for handling uncertainty, and robust stability in multiple, input multiple, output (MIMO) systems introduced a formulation for design under uncertainty in input parameters, which utilizes a smooth nonlinear program that approximates the feasibility problem. Androulakis et al. [19] investigated the effect of uncertainty in the microscopic reaction rate constants on macroscopic observables such as autoignition delay. Samsatli [20] introduced a robustness metric for optimization under parametric uncertainty. Using a similar example, Skogestad and Morari [16] compare the differences between the use of SSV's for SISO and MIMO systems and present an extension of the Nyquist stability criterion for MIMO systems. This approach will be used in the rest of the current work. In essence, this approach utilizes the Nyquist plots shown in Figures 4 and 5 to capture the impact of parametric uncertainty on the performance of the system. Figure 6(a) focuses on the response at $1 \mathrm{mHz}$ when several 


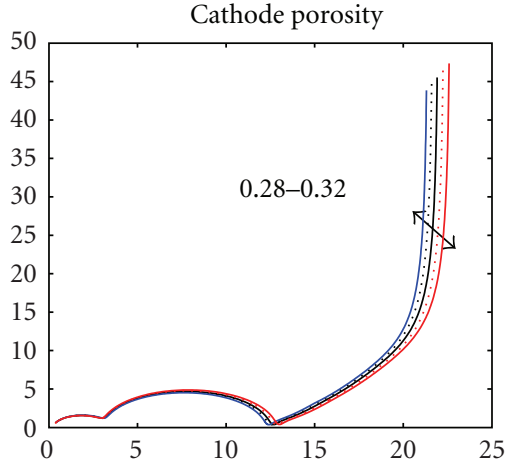

(a)

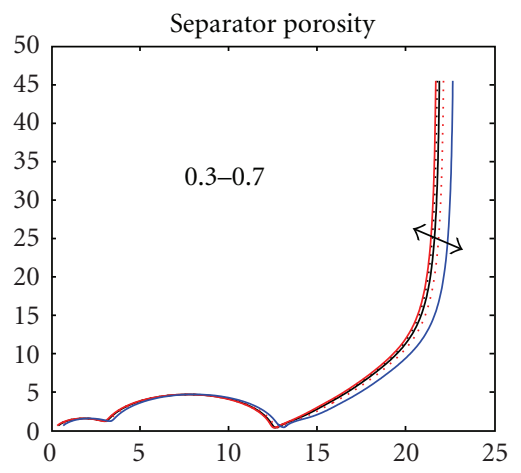

(d)

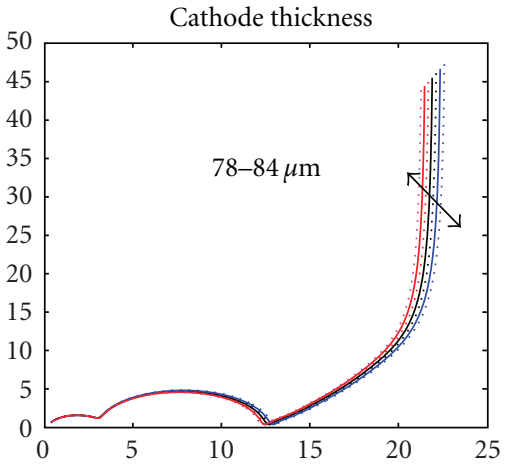

(b)

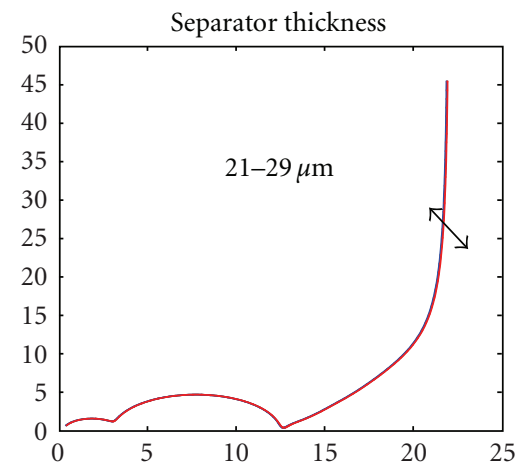

(e)

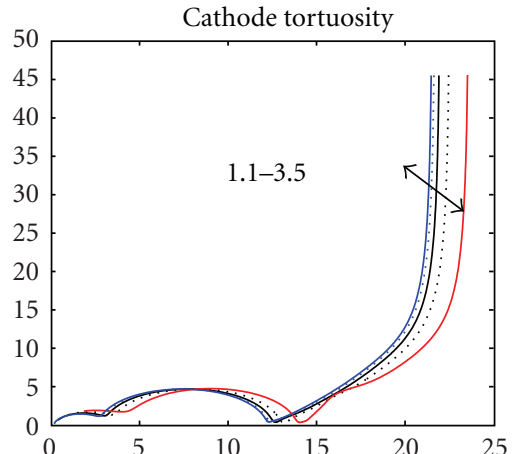

(c)

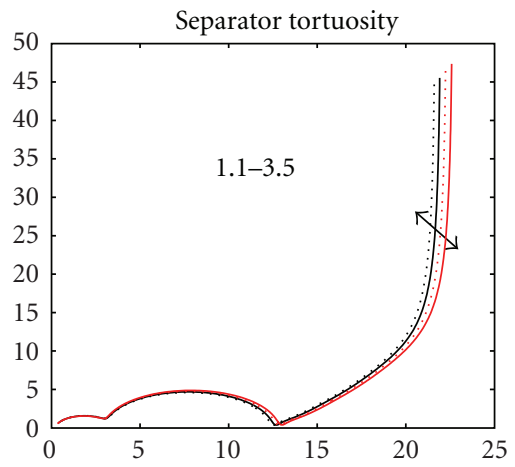

(f)

FIgure 5: Comparison of the sensitivity of the cell impedance to the corresponding design parameters within the cathode versus the separator; for the choice of nominal parameters shown in Table 1, the variations on the separator do not impact the cell performance as much as those of the cathode.

parameters like the cathode porosity, particle size, and thickness are perturbed; these are results super-imposed from Figure 4. The method proposed by Skogestad then approximates the uncertainty in the response by a disc whose radius spans the uncertainty in the input space. Corresponding to each of the parameters shown in the figure, there exists a separate disc-approximation, as shown in Figure 6(b). In practice, the discs may not be circles for all cases; however, identifying the shape of the actual uncertain region in the response space can be a cumbersome task for the case with multiple parameters, and the methodology shown in the figure is a good approximation. The use of the individual disc-approximation corresponds to the design of experiments optimizing one parameter at a time as described in the previous sections. In the case shown in Figure 6, for example, the use of individual circles to optimize performance represents adjusting the porosity distribution, particle sizes, and variations in electrode thickness, one at a time. In practice, these parameters can be tuned individually to accomplish different goals, changing one of these parameters often leads to a change in the others-making the tuning process more complicated than that for an SISO system. The worst case scenario is represented by the circumcircle of the individual confidence regions. The best possible scenario for a given set of parameters connects the centers of the confidence discs; for this case, there is no uncertainty in the value of any parameter; that is, each parameter is held at its nominal value and the impedance response collapses to a point value as well.

The representation of the uncertainty from the various parameters simultaneously, as shown in Figure 6, has several advantages. To begin with, one can readily see that the sensitivity of the circumcircle to the various parameters is a function of the nominal values for the parameters as well as the frequency of choice. In other words, the individual sensitivities shown in Figures 4 and 5 can now be compared against one another, by choosing an appropriate frequency. In the example shown in Figure 6, the particle size has the largest impact (i.e., the disc corresponding to that parameter has the largest diameter) on the fluctuations in the impedance, and variations in the thickness of the electrode may be masked by those in the particle size distribution within the scope of the electrochemical model used in this study. Subsequently, any effort to improve the thickness of the electrode will not lead to fruition, until the variations in the particle size are addressed for the nominal values of parameters used here. Secondly, comparison of the effect of variations in the individual parameters provides an easy-to-use metric as opposed to the variations themselves; it reduces the need for arbitrary specifications on the individual parameters or that for an extensive design of experiments, since one measure (here the cell impedance at a preset frequency) is used to 


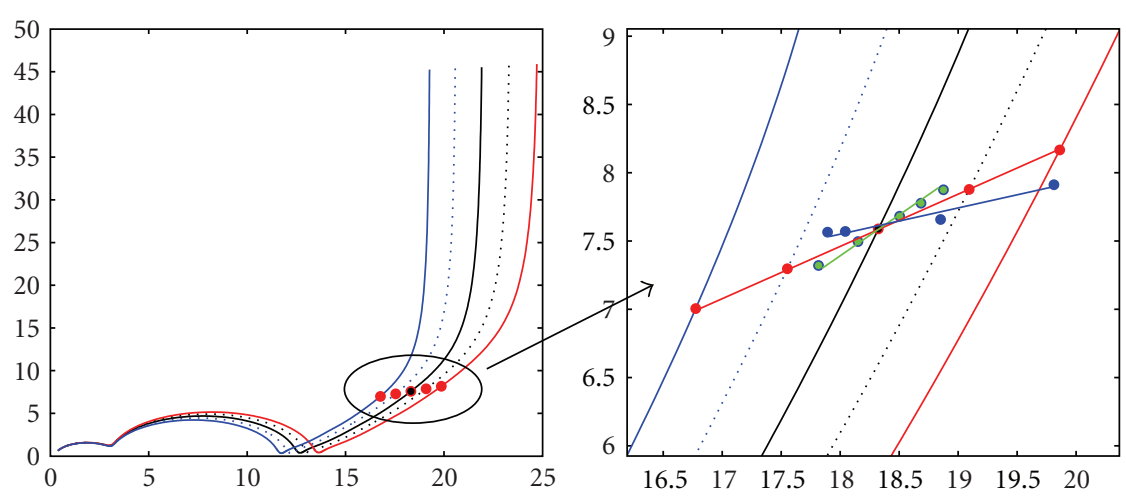

(a)

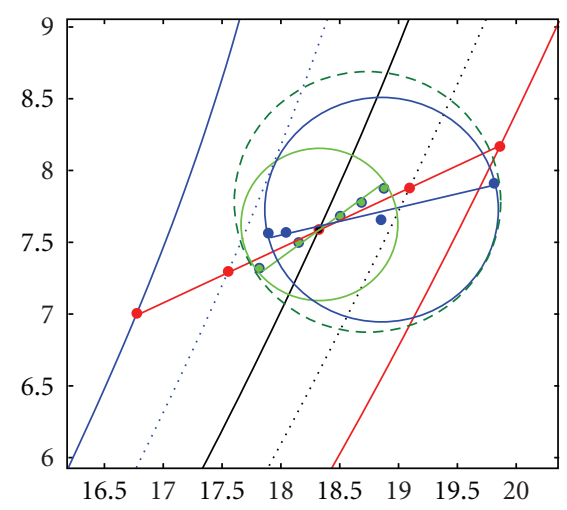

(c)

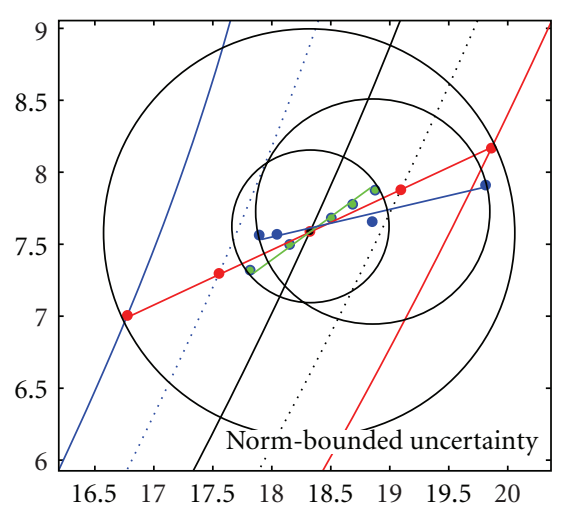

(b)

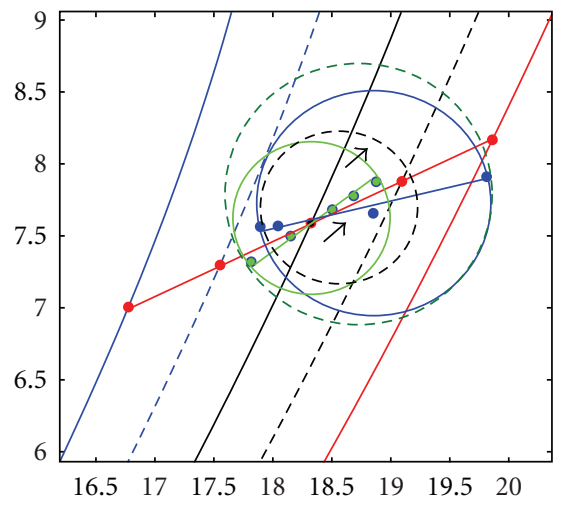

(d)

FIgURE 6: (a) Superimposing sensitivity data from Figures 4 and 5. (b) Construction of the norm-bounded uncertainties for the individual parameters: the smallest disc corresponds to the uncertainty in the cathode thickness, the medium-sized disc to that of the cathode porosity, and the largest to the particle size within the cathode. (c) Determining the overall uncertainty in the impedance response at $1 \mathrm{mHz}$ : the dashed circumcircle shows the uncertainty region covering the influence of the variations in the individual parameters. (d) Setting the nominal value for the cathode thickness at $75 \mu \mathrm{m}$ instead of $70 \mu \mathrm{m}$ moves the individual uncertainty disc from the solid circle shown in (c) to the dotted circle, and the overall uncertainty from the dashed circumcircle to coincide with the individual disc corresponding to that of the porosity variations.

assess the impact of variations in each parameter. All efforts to minimize the cell-to-cell variations are in essence attempts to minimize the radius of the circumcircle shown in Figure 6. The obvious path forward, is to minimize the variations in the individual parameters; however, it is not always economical to tune each parameter to the desired level of uniformity. One typical example is the thickness of the current collectors versus that of the active material or the separator described in the previous section. In this case, an alternate choice of the nominal values for the different parameters may help in reducing the impact of variations in the parameter that is more difficult to tune. For instance, adjusting the nominal value for the separator thickness such that any variation on this parameter falls within the observable range of impedance variations due to the other parameters will greatly reduce the efforts that may otherwise go towards unachievable thickness uniformity improvements on a $25 \mu \mathrm{m}$ thin polymer film, to bring it at par with a $25 \mu \mathrm{m}$ aluminum foil. Figure 6(d) shows another example of this approach, where two parameters (the thickness and porosity of the cathode) are considered; for the nominal values used, the two small discs shown in Figure 6(a) represent the variability in the cell impedance. However, shifting the nominal thickness of the cathode by $5 \mu \mathrm{m}$ effectively moves the uncertainty due to the thickness into the range corresponding to that from the porosity.

In essence, we propose the following steps to translate requirements on cell-to-cell uniformity to tolerance specifications for the component manufacturers: (i) obtain the acceptable range of impedance values for the cells from different lots based on the end-user requirements, operating conditions, and so forth, (ii) use this range as the diameter for the circumcircle described in Figure 6, (iii) obtain the experimentally measured distributions in the values of the properties of interest in the different components (anode, foil, separator, electrolyte, etc.) to construct the individual confidence intervals, and (iv) match the variations in the components to accommodate all the subcircles within the acceptable range of impedance values as given by the circumcircle.

The choice of frequency domain for such analysis is based on the ease of detecting the sensitivity of the response to 


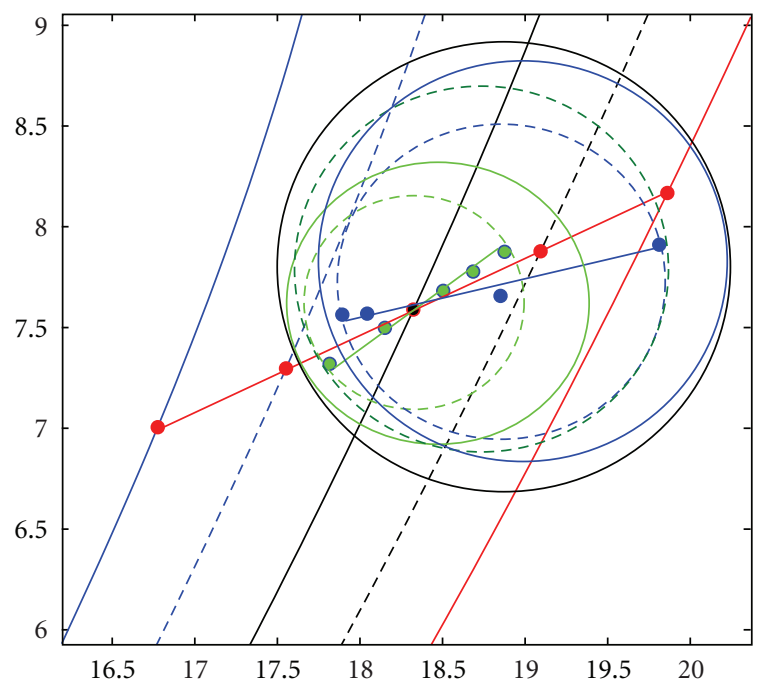

FIGURE 7: Effect of temperature on the sensitivity of the impedance response to cathode thickness and porosity: the dotted circles correspond to the uncertainties at room temperature $(298 \mathrm{~K})$ and the solid circles correspond to equivalent values at low temperature (263 K).

changes in the different parameters. A similar procedure using time-domain data (e.g., discharge curves) can be developed; however, it is usually difficult to distinguish experimental noise from subtle changes due to variations in the parameters. Hence, the time domain data do not reveal such trends until after prolonged use of the battery. A second advantage is the possibility of identifying a range of frequencies depending on the time constants, for the choice of parameters investigated. Also, the conventional tools used in stability analyses and robust design [13-23] can be readily extended to battery design, by posing the deviations out of the prespecified tolerance limit as being thrust into an unstable region.

3.4. Parameter Tuning Based on Operating Conditions. The previous subsections illustrate how the different parameters influence the variations within the cell to different extents. A lot of these variations also depend on the operating conditions the battery is subjected to. Figure 7 illustrates the effect of variations in the same set of parameters shown in Figure 6 at a lower temperature. As reported in the literature, the transport properties of the electrolyte are very different from those at room temperature. As a result, the sensitivity of the cell impedance to parameters like the porosity and tortuosity of the electrode is increased, and the uncertainty in the cell impedance is magnified. As seen in Figure 7, the effect of the cathode porosity is comparable with the particle-size effects when the battery is operated at low temperatures. Hence if the battery operates for a majority of the time at lower temperatures, tailoring the design to accommodate this finding will lead to addressing low temperature limitations better.

\section{Summary}

Cell-to-cell variations are often attributed to uncertainties in the design parameters for the different cell components. Knowing the sensitivity of the cell performance to the parameters of interest helps battery manufacturers to focus on the right set of steps, instead of enforcing ad-hoc criteria on the tolerances for different properties at quality check. A methodology was presented to facilitate studying the relative effects of multiple parameters simultaneously and to prioritize testing procedure during design of experiment studies. The process outlined above may be used to check if the individual cells from different lots conform to a prespecified tolerance limit for the battery, by measuring the scatter in the a.c. impedance of the cells at a suitable frequency. Finally, the simulation results for the different operating conditions will help tailor the design parameters according to the targeted end use. A few open questions remain: the results presented here depend entirely on the model of choice; no allowance has been provided in this work to accommodate for unmodeled dynamics. There are accounts of representing such instances as uncertainties as well; however, in most practical instances, it is hard to distinguish between variations in the parameters and those from under represented physical phenomena. A rigorous procedure to determine the "optimal" frequency at which the sensitivity analysis should be carried out has not been developed yet, for the case with uncertain parameters. The approximation of the uncertain region by discs as discussed earlier has been questioned by Bequette [2], since this approach amplifies the actual margin of discrepancy and introduces purely hypothetical scenarios in some cases. Physics-based definition of uncertainties has been recommended instead; however, the implementation requires a rigorous formulation, hence, we did not adopt this approach here. However, this idea warrants further exploration in subsequent analyses.

\section{References}

[1] D. Linden and T. B. Reddy, Eds., Handbook of Batteries, 3rd edition, 2003.

[2] Electric and Hybrid Vehicle Propulsion Battery System Safety Standard, SAE J2929.

[3] E. Barsoukov and J. R. McDonald, Eds., Impedance Spectroscopy: Theory, Experiment, and Applications, Wiley-Interscience, Hoboken, NJ, USA, 2nd edition, 2005.

[4] R. Darling and J. Newman, "Modeling a porous intercalation electrode with two characteristic particle sizes," Journal of the Electrochemical Society, vol. 144, no. 12, pp. 4201-4208, 1997.

[5] J. P. Meyers, M. Doyle, R. M. Darling, and J. Newman, "Impedance response of a porous electrode composed of intercalation particles," Journal of the Electrochemical Society, vol. 147, no. 8, pp. 2930-2940, 2000.

[6] D. Dees, E. Gunen, D. Abraham, A. Jansen, and J. Prakash, "Alternating current impedance electrochemical modeling of lithium-ion positive electrodes," Journal of the Electrochemical Society, vol. 152, no. 7, pp. A1409-A1417, 2005.

[7] D. E. Stephenson, E. M. Hartman, J. N. Harb, and D. R. Wheeler, "Modeling of particle-particle interactions in porous cathodes for lithium-ion batteries," Journal of the Electrochemical Society, vol. 154, no. 12, pp. A1146-A1155, 2007. 
[8] G. Sikha and R. E. White, "Analytical expression for the impedance response of an insertion electrode cell," Journal of the Electrochemical Society, vol. 154, no. 1, pp. A43-A54, 2007.

[9] G. Sikha and R. E. White, "Analytical expression for the impedance response for a lithium-ion cell," Journal of the Electrochemical Society, vol. 155, no. 12, pp. A893-A902, 2008.

[10] G. S. Nagarajan, J. W. Van Zee, and R. M. Spotnitz, "A mathematical model for intercalation electrode behavior: I. Effect of particle-size distribution on discharge capacity," Journal of the Electrochemical Society, vol. 145, no. 3, pp. 771-779, 1998.

[11] R. N. Methekar, P. W. C. Northrop, K. Chen, R. D. Braatz, and V. R. Subramanian, "Kinetic Monte Carlo simulation of surface heterogeneity in graphite anodes for lithium-ion batteries: passive layer formation," Journal of The Electrochemical Society, vol. 158, pp. A363-A368, 2011.

[12] S. Motupally, C. C. Streinz, and J. W. Weidner, "Proton diffusion in nickel hydroxide films measurement of the diffusion coefficient as a function of state of charge," Journal of the Electrochemical Society, vol. 142, no. 5, pp. 1401-1408, 1995.

[13] G. E. P. Box, "Fitting empirical data," Annals of the New York Academy of Sciences, vol. 86, pp. 792-794, 1960.

[14] S. Santhanagopalan and R. E. White, "Modeling parametric uncertainty using polynomial chaos theory," ECS Transactions, vol. 3, no. 27, pp. 243-256, 2007.

[15] W. L. Luyben, Process Modeling, Simulation and Control for Chemical Engineers, McGraw-Hill, New York, NY, USA, 2nd edition, 1989.

[16] S. Skogestad and M. Morari, "Model uncertainty, process design, and process control," in Proceedings of the AIChE Annual Meeting, Chicago, Ill, USA, November, 1985.

[17] S. Skogestad and I. Postlethwaite, Multivariable Feedback Control: Analysis and Design, John Wiley \& Sons, West Sussex, UK, 2nd edition, 2005.

[18] W. C. Rooney and L. T. Biegler, "Optimal process design with model parameter uncertainty and process variability," AIChE Journal, vol. 49, no. 2, pp. 438-449, 2003.

[19] I. P. Androulakis, J. M. Grenda, T. A. Barckholtz, and J. W. Bozzelli, "Propagation of uncertainty in chemically activated systems," AIChE Journal, vol. 52, no. 9, pp. 3246-3256, 2006.

[20] N. J. Samsatli, L. G. Papageorgiou, and N. Shah, "Robustness metrics for dynamic optimization models under parameter uncertainty," AIChE Journal, vol. 44, no. 9, pp. 1993-2006, 1998.

[21] S. Balakrishnan, P. Georgopoulos, I. Banerjee, and M. Ierapetritou, "Uncertainty considerations for describing complex reaction systems," AIChE Journal, vol. 48, no. 12, pp. 2875$2889,2002$.

[22] K. A. McDonald, A. Palazoglu, and B. W. Beuette, "Impact of model uncertainty descriptions for high-purity distillation control," AIChE Journal, vol. 34, no. 12, pp. 1996-2004, 1988.

[23] B. A. Ogunnaike and W. H. Ray, Process Dynamics, Modeling, and Control (Topics in Chemical Engineering), Oxford University Press, Cambridge, Mass, USA, 1994.

[24] C. Y. Wang and V. Srinivasan, "Computational battery dynamics (CBD)-electrochemical/thermal coupled modeling and multi-scale modeling," Journal of Power Sources, vol. 110, no. 2, pp. 364-376, 2002.

[25] M. Dubarry, N. Vuillaume, and B. Y. Liaw, "From single cell model to battery pack simulation for Li-ion batteries," Journal of Power Sources, vol. 186, no. 2, pp. 500-507, 2009.
[26] Y.-B. Yi, C.-W. Wang, and A. M. Sastry, "Two-dimensional vs. three-dimensional clustering and percolation in fields of overlapping ellipsoids," Journal of the Electrochemical Society, vol. 151, no. 8, pp. A1292-A1300, 2004. 


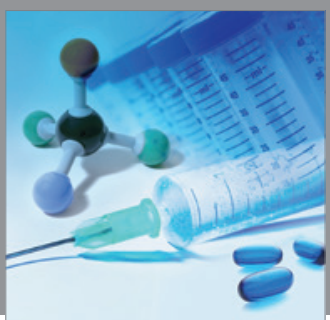

International Journal of

Medicinal Chemistry

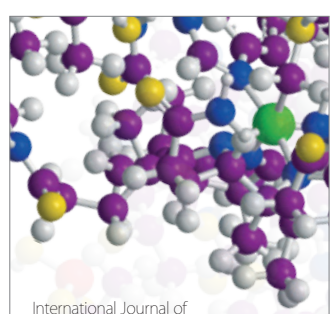

Carbohydrate Chemistry

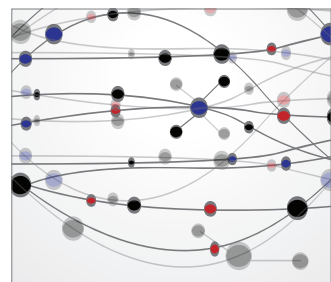

The Scientific World Journal
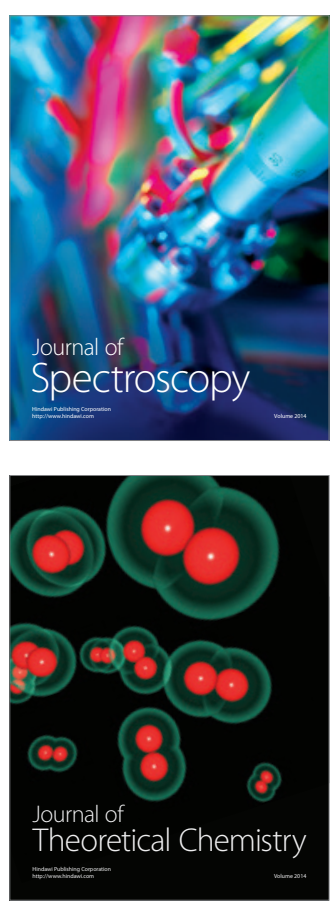
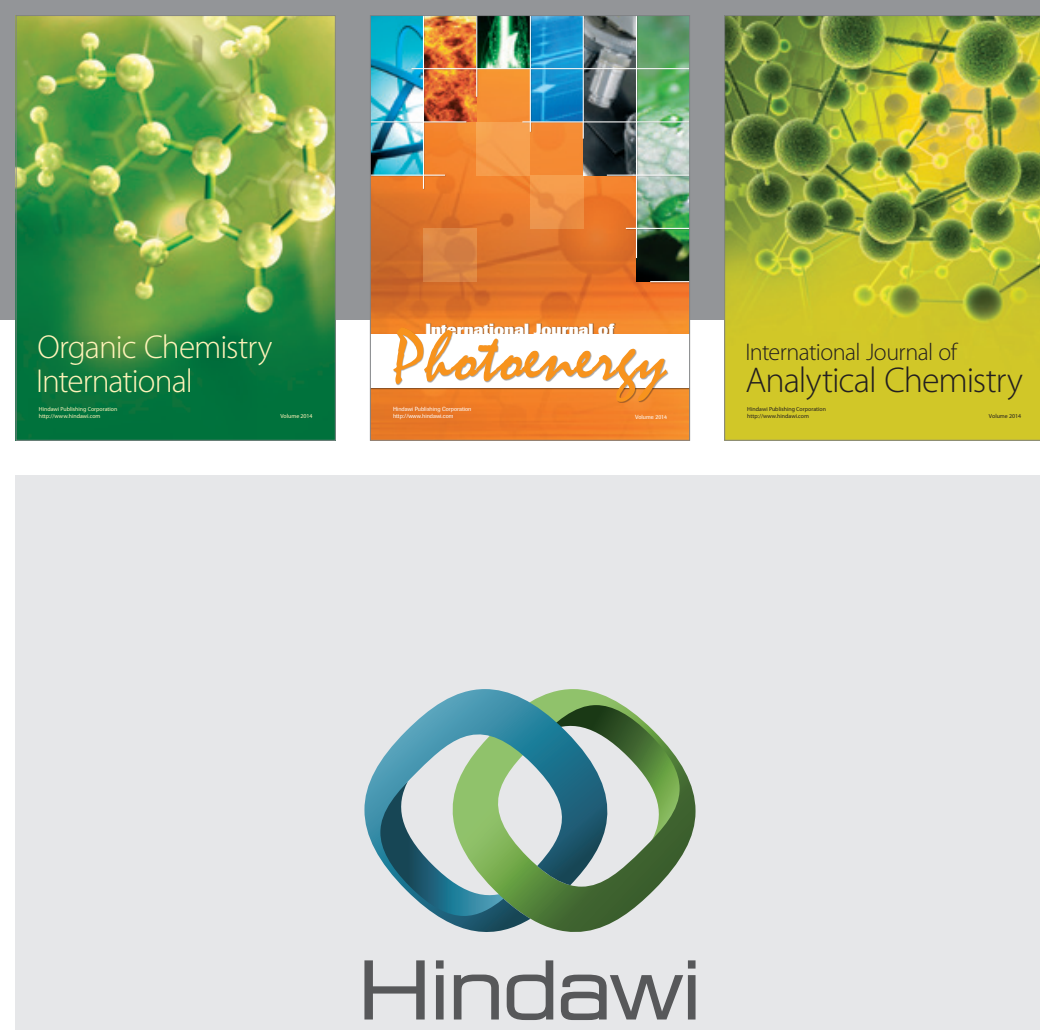

Submit your manuscripts at

http://www.hindawi.com
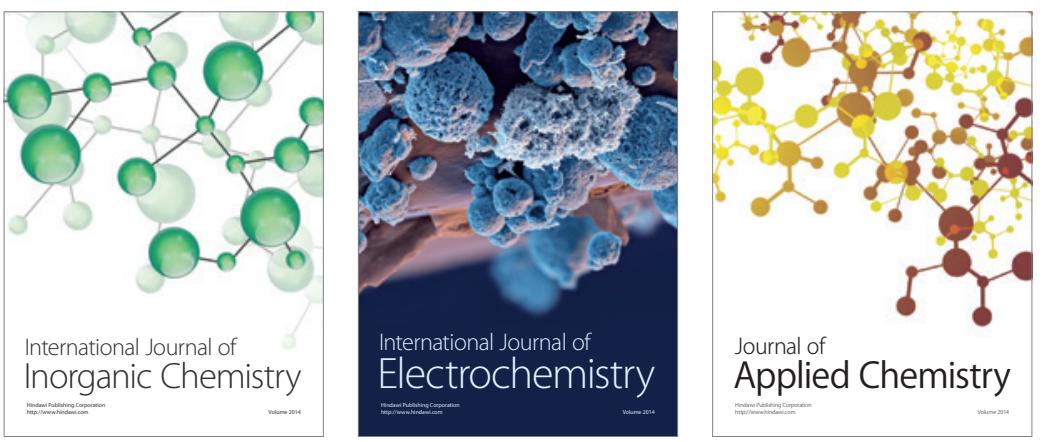

Journal of

Applied Chemistry
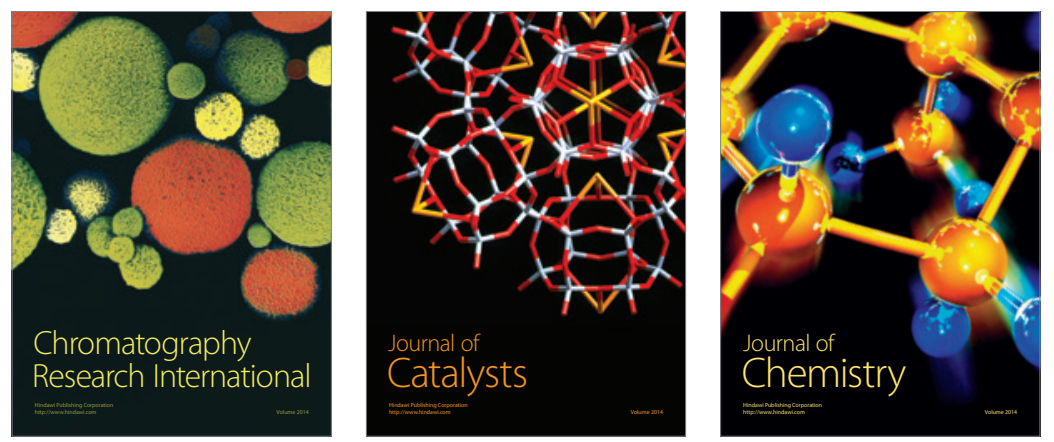
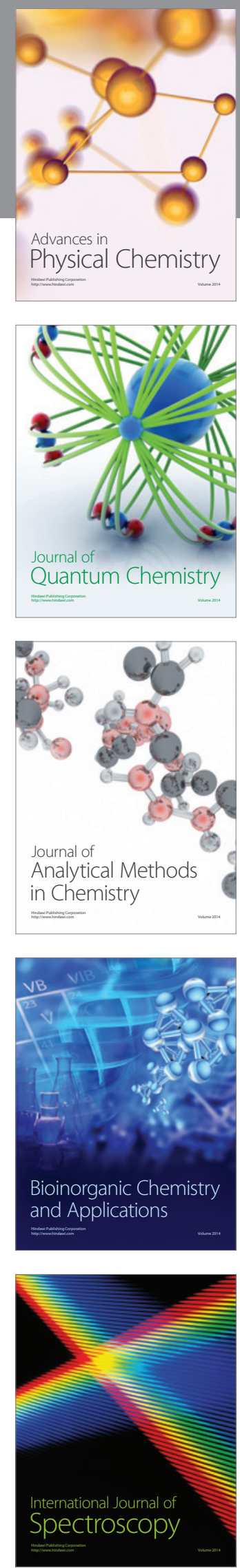